\title{
Notas sinonímicas em Lepturini sul-americanos (Coleoptera, Cerambycidae, Lepturinae)
}

\author{
Miguel A. Monné1,3, Marcela L. Monné ${ }^{2,4}$ \& Ubirajara R. Martins ${ }^{2,3}$
}

\begin{abstract}
${ }^{1}$ Museu Nacional, Universidade Federal do Rio de Janeiro. Quinta da Boa Vista, São Cristóvão, 20940-040 Rio de Janeiro-RJ, Brasil. ${ }^{2}$ Museu de Zoologia, Universidade de São Paulo. Caixa Postal 42494, 04218-970 São Paulo-SP, Brasil.

${ }^{3}$ Pesquisador $\mathrm{CNPq}$.

${ }^{4}$ Bolsista FAPESP.
\end{abstract}

\begin{abstract}
Aвstract. Synonymical notes on South American Lepturini (Coleoptera, Cerambycidae, Lepturinae). New synonyms proposed: Strangalia flavocincta (Thomson, 1860) $=$ Ophistomis tristis Melzer, 1922 syn. nov. $=$ O. latifasciata Melzer, 1926 syn. nov.; Strangalia succincta (Redtenbacher, 1867) $=$ O. auriflua Redtenbacher, 1867 syn. nov.; Strangalia melanura $($ Redtenbacher, 1867) $=$ Euryptera dimidiata Redtenbacher, 1867 syn. nov.; Strangalia lyrata $($ Redtenbacher, $1867)=$ Ophistomis discophora Redtenbacher, 1867 syn. nov.; Strangalia fulvicornis (Bates, 1872) = Ophistomis variabilis Melzer, 1926 syn. nov. $=$ O. flavovittata Melzer, 1926 syn. nov.; Strangalia melanophthisis (Berg, 1889) reval. = Euryptera melanura var. nigripennis Melzer, 1930 syn. nov.; Anastrangalia sanguinolenta (Linnaeus, 1761) (species introduced in Argentina) $=$ Leptura bonaeriensis Burmeister, 1865 syn. nov.
\end{abstract}

KEYwords. Cerambycidae; Coleoptera; Lepturinae; new synonymies.

Resumo. Novas sinonímias propostas: Strangalia flavocincta (Thomson, 1860) = Ophistomis tristis Melzer, 1922 syn. nov. $=$ O. latifasciata Melzer, 1926 syn. nov.; Strangalia succincta (Redtenbacher, 1867) $=$ O. auriflua Redtenbacher, 1867 syn. nov.; Strangalia melanura (Redtenbacher, 1867) = Euryptera dimidiata Redtenbacher, 1867 syn. nov.; Strangalia lyrata (Redtenbacher, 1867) = Ophistomis discophora Redtenbacher, 1867 syn. nov.; Strangalia fulvicornis $($ Bates, 1872) $=$ Ophistomis variabilis Melzer, 1926 syn. nov. $=$ O. flavovittata Melzer, 1926 syn. nov.; Strangalia melanophthisis $($ Berg, 1889) reval. = Euryptera melanura var. nigripennis Melzer, 1930 syn. nov.; Anastrangalia sanguinolenta (Linnaeus, 1761) (espécie introduzida na Argentina) = Leptura bonaeriensis Burmeister, 1865 syn. nov.

Palavras-Chave. Cerambycidae; Coleoptera; Lepturinae; novas sinonímias.

A tribo Lepturini compreende seis gêneros e 35 espécies na América do Sul, dos quais três gêneros e 31 espécies ocorrem no Brasil (MonNÉ 1995). Com a finalidade de iniciar a revisão dos Lepturini sul-americanos, examinamos abundante material das espécies distribuídas na Floresta Atlântica, o que possibilitou propor diversas sinonímias. De seis espécies de Strangalia Audinet-Serville, 1835, examinamos 650 exemplares, o que permitiu detectar as variações cromáticas dentro de cada táxon, que ocorrem independentemente em diversas localidades. Ambos os sexos das espécies de Strangalia distribuídas no leste e sudeste do Brasil variam consideravelmente na coloração e, ao mesmo tempo, esta variabilidade cromática é diferente nos dois sexos de cada espécie.

O material mencionado pertence às instituições: MACN, Museo Argentino de Ciencias Naturales "Bernardino Rivadavia", Buenos Aires; MNHN, Muséum National d'Histoire Naturelle, Paris; MNRJ, Museu Nacional, Universidade Federal do Rio de Janeiro, Rio de Janeiro; MZSP, Museu de Zoologia, Universidade de São Paulo, São Paulo.

O material-tipo das espécies descritas por ReDTENBACHER (1867) não foi examinado, segundo HoRN \& KAHLE (1936: 220) os espécimens estão depositados no Naturhistorisches Museum, Viena, Austria. Contudo, as descricões originais e figuras permitiram a identificação das espécies.
Strangalia flavocincta (Thomson, 1860)

(Figs. 1-6)

Ophistomis flavocinctus Thomson, 1860: 155; 1864: 140; Lacordaire, 1869: 451; Zajciw, 1970: 229, figs 1, 2.

Euryptera flavocincta; Thomson, 1878: 5 (tipo).

Strangalia flavocincta; Linsley \& Chemsak, 1971: 24; Zajciw, 1974: 42 (distr.); Monné, 1995: 63 (cat.).

Ophistomis tristis Melzer, 1922: 5; 1927: 160; Zikán \& Wygodzinsky, 1948: 41 (tipo); Bachmann \& Di Iorio, 2002: 83 (tipos). Syn. nov.

Ophiostomis [sic] tristis; Zikán \& Zikán, 1944: 12 (distr.).

Strangalia tristis; Linsley \& Chemsak, 1971: 24; Zajciw, 1974: 42 (distr.); Monné, 1995: 69 (cat.).

Ophistomis latifasciata Melzer, 1926: 9; Zikán \& Wygodzinsky, 1948: 40 (tipo); Zajciw \& Seabra, 1968: 70 (distr.); Zajciw, 1972: 47 (distr.). Syn. nov.

Ophiostomis [sic] latifasciata; Zikán \& Zikán, 1944: 13 (distr.).

Strangalia latifasciata; Linsley \& Chemsak, 1971: 24; Monné, 1995: 65 (cat.).

Examinamos o holótipo de Ophistomis flavocinctus, proveniente do Brasil (MNHN) e os holótipos de Ophistomis tristis e de O. latifasciata (MZSP), procedentes, respectivamente, de Passa Quatro, Minas Gerais e Itatiaia, Rio de Janeiro.

Espécie muito variável na coloração; nos machos, prevalece nos élitros ou a cor preta ou castanho-alaranjada, 
com ou sem manchas castanho-escuras e pernas pretas ou vermelhas (Figs. 1-3). Nas fêmeas, os élitros são amareloalaranjados com uma a três manchas transversais pretas ou totalmente pretos e os fêmures pretos ou vermelhos (Figs. 4$6)$.

Material examinado. BRASIL, fêmea (holótipo de O. flavocinctus) (MNHN). Bahia: Encruzilhada (Motel da Divisa, Estrada Rio-Bahia km 965), 3 machos, 4 fêmeas (MNRJ). Espírito Santo: Barra de São Francisco (Córrego do Itá), macho, 2 fêmeas; Colatina, fêmea; Linhares, 4 machos, 4 fêmeas; (Parque Sooretama), 5 machos, 7 fêmeas (MNRJ). Minas Gerais: Passa Quatro, macho (holótipo de O. tristis) (MZSP); Pedra Azul, 5 machos, 5 fêmeas; Santana do Riacho (Parque Nacional Serra do Cipó), 2 machos (MNRJ). Rio de Janeiro: Barra de São João, 7 machos, 2 fêmeas; Itatiaia $(700 \mathrm{~m})$, fêmea (holótipo de $O$. latifasciata) (MZSP). Rio de Janeiro (Corcovado), macho; (Floresta da Tijuca), 3 machos, 3 fêmeas (MNRJ). São Paulo: Peruibe, fêmea; São Paulo (Jabaquara), 6 machos, 8 fêmeas (MNRJ).

Strangalia melanura (Redtenbacher, 1867) (Figs. 7-9)

Euryptera melanura Redtenbacher, 1867: 189; Belon, 1897: 341; Melzer, 1930: 190; Zikán \& Zikán, 1944: 13 (distr.); Baucke, 1957: 23; Buck, 1959: 587 (distr.); Zajciw \& Seabra, 1968: 70 (distr.); Zajciw, 1972: 48 (distr.); Monné, 1995: 56 (cat.).

Strangalia melanura; Di Iorio, 1998: 143, fig. 14

Euryptera dimidiata Redtenbacher, 1867: 189; Belon, 1897: 341; Gounelle, 1911: 5 (distr.); Bruch, 1912: 193 (cat.); Zikán \& Zikán, 1944: 13 (distr.); Baucke, 1957: 22; Buck, 1959: 587 (distr.); Zajciw \& Ruffinelli, 1962: 18 (distr.); Zajciw \& Seabra, 1968: 70 (distr.); Monné \& Zajciw, 1970: 31 (distr.); Zajciw, 1972: 48 (distr.); Monné, 1995: 55 (cat.).; Mecke et al., 2000: 169 (hosp.); Monné, 2002: 40 (hosp.). Syn. nov.

Strangalia dimidiata; Di Iorio, 1998: 141, fig. 2; Mecke, 2002: 30, fig. 39 (hosp.).

Strangalia melanura e $S$. dimidiata foram descritas por REDTENBACHer (1867) do Rio de Janeiro. A coloração elitral varia em ambos o sexos, desde a metade basal com o tegumento amarelo a totalmente preto.

Material examinado. BRASIL. Minas Gerais: Maria da Fé, fêmea; Poços de Caldas, 6 machos, 3 fêmeas; Santana do Riacho (Parque Nacional Serra do Cipó), 11 machos, 3 fêmeas; Virginia, macho, 2 fêmeas. Rio de Janeiro: Itatiaia, 4 fêmeas; Petrópolis, macho; Rio de Janeiro (Corcovado), macho; (Floresta da Tijuca), 2 machos, 3 fêmeas; (Represa Rio Grande), macho, fêmea. São Paulo: Amparo, macho; São Bernardo do Campo, macho; São José Barreiro (Serra da Bocaina), 101 machos, 37 fêmeas; São Paulo (Jabaquara), 2 machos, 3 fêmeas. (MNRJ).

\section{Strangalia succincta (Redtenbacher, 1867)}

(Figs. 10-13)

Ophistomis succincta Redtenbacher, 1867: 190, pr. 5, fig. 8. Strangalia succincta; Monné, 1995: 68 (cat.).

Ophistomis auriflua Redtenbacher, 1867: 190, pr. 5, fig. 9. Syn. nov. Strangalia auriflua; Zajciw, 1974: 42 (distr.); Monné, 1995: 60 (cat.).

Ophistomis succinta e $O$. auriflua foram descritas do Rio de Janeiro. Pelas descrições e figuras, ambos os nomes correspondem a exemplares fêmeas que variam consideravelmente na coloração: pronoto totalmente amarelo ou com mácula centro-mediana preta ou castanho-escura. A coloração elitral oscila entre predominantemente preta com máculas umerais e medianas amarelas a predominantemente amarela com orla basal e ápices pretos (Figs. 10-12). Nos machos, de coloração mais constante, o pronoto é amarelo com mancha centro-mediana e os élitros são pretos, exceto úmeros alaranjados (Fig. 13).

Material examinado. BRASIL. Bahia: Barrolandia, fêmea; Una, fêmea. Espirito Santo: Linhares, macho; (Parque Sooretama), macho, fêmea; Mun. São Francisco (Córrego do Itá), fêmea. Rio de Janeiro: Barra de São João, 13 machos; Rio de Janeiro (Floresta da Tijuca), 2 machos. São Paulo: Indiana, fêmea; Marília, macho; Teodoro Sampaio, 3 fêmeas; Vale do Rio Pardo, fêmea. Paraná: Rolandia, macho, fêmea. (MNRJ).

Strangalia lyrata (Redtenbacher, 1867) (Figs. 14-15)

Ophistomis lyrata Redtenbacher, 1867: 190; Buck, 1959: 587 (distr.); Zajciw, 1972: 48 (distr.).

Ophiostomis [sic] lyrata; Zikán \& Zikán, 1944: 13 (distr.).

Strangalia lyrata; Monné, 1995: 66 (cat.).

Ophistomis discophora Redtenbacher, 1867: 191; Zajciw, 1958: 10 (distr.); 1972: 47 (distr.). Syn. nov.

Ophiostomis [sic] discophora; Zikán \& Zikán, 1944: 12 (distr.).

Strangalia discophora; Zajciw, 1974: 42 (distr.); Monné, 1995: 62 (cat.).

Pelas descricões originais observou-se que REDTENBACHER (1867) descreveu, do Rio de Janeiro, o macho (O. lyrata) e a fêmea (O. discophora) da mesma espécie. A coloração apresenta ligeiras variações nos machos, com élitros enegrecidos, restando a orla basal e pequena mácula anteapical amarelas (Fig. 14). Nas fêmeas, os élitros são alaranjados com três faixas transversais pretas (Fig. 15).

Material examinado. BRASIL. Rio de Janeiro: Nova Friburgo, fêmea; Petrópolis, 2 machos, 4 fêmeas; Rio de Janeiro (Corcovado), 12 machos, 8 fêmeas; (Floresta da Tijuca), 4 machos, 2 fêmeas. São Paulo: Peruibe, macho; São Paulo (Cantareira), macho. (MNRJ).

\section{Strangalia fulvicornis (Bates, 1872)} (Figs. 16-17)

Ophistomis fulvicornis Bates, 1872: 183; Buck, 1959: 587 (distr.); Monné \& Zajciw, 1970: 29 (distr.).

Ophiostomis [sic] fulvicornis; Gounelle, 1911: 105.

Strangalia fulvicornis; Linsley \& Chemsak, 1971: 24; Zajciw, 1974: 42 (distr.); Monné, 1995: 64 (cat.); Di Iorio, 1998: 143, 144 (chave).

Ophistomis variabilis Melzer, 1926: 8; Zikán \& Wygodzinsky, 1948: 41 (tipo); Zajciw, 1972: 48 (distr.). Syn. nov.

Ophiostomis [sic] variabilis; Zikán \& Zikán, 1944: 13 (distr.).

Strangalia variabilis; Linsley \& Chemsak, 1971: 24; Monné, 1995: 69 (cat.).

Ophistomis flavovittata Melzer, 1926: 10; Zikán \& Wygodzinsky, 1948: 40 (tipo); Zajciw \& Seabra, 1968: 70 (distr.). Syn. nov.

Strangalia flavovittata; Linsley \& Chemsak, 1971: 24 (sin.); Monné, 1995: 64 (cat.).

Ophistomis rustica Melzer, 1926: 10; 1930: 190; Zikán \& Wygodzinsky, 1948: 41 (tipo); Zajciw \& Seabra, 1968: 70 (distr.).

Ophiostomis [sic] rustica; Zikán \& Zikán, 1944: 13 (distr.). 

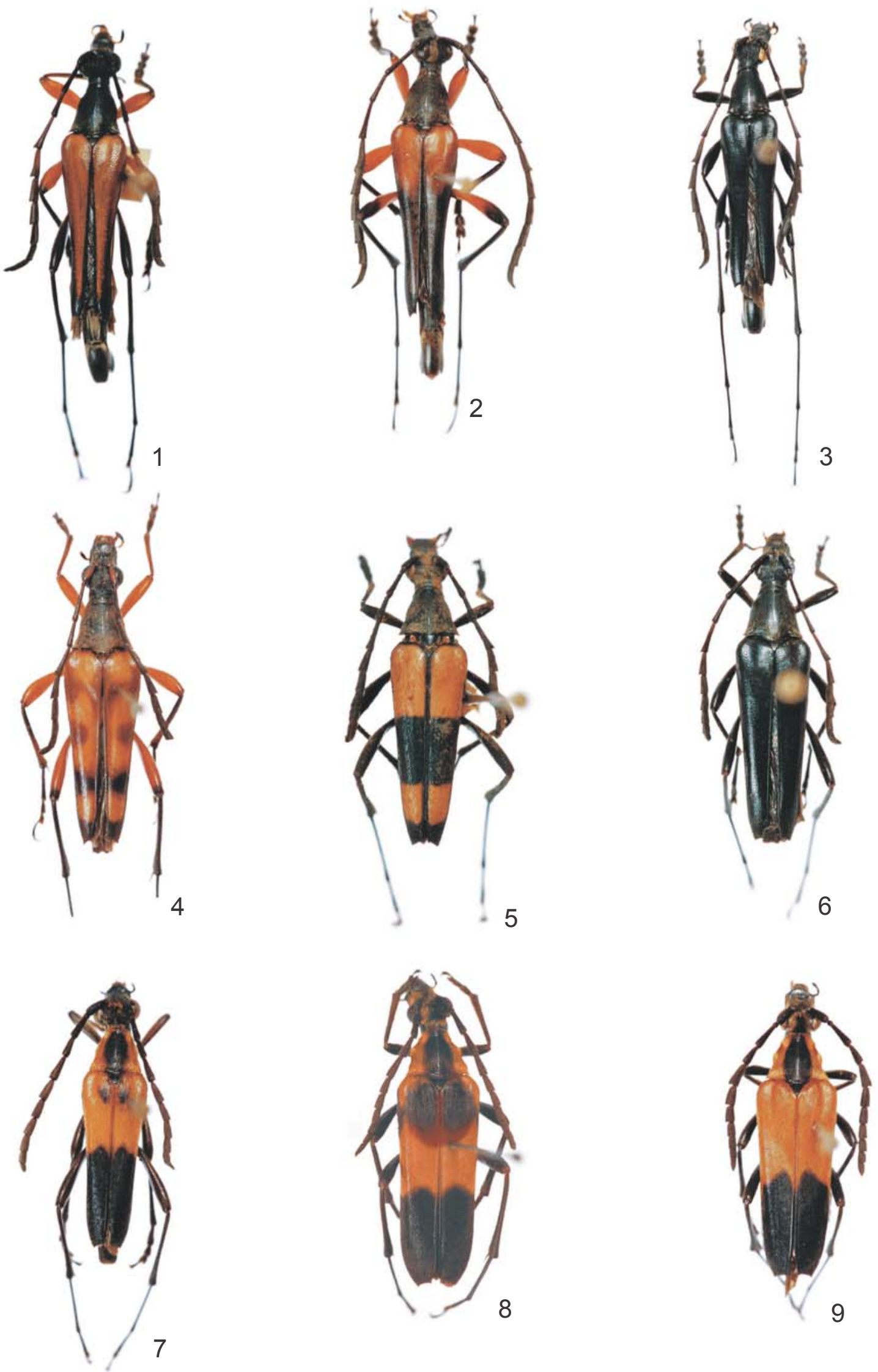

Figs. 1-9. 1-6, Strangalia flavocincta: 1, macho, comprimento, $17,2 \mathrm{~mm} ; \mathbf{2}$, macho, $19,3 \mathrm{~mm} ; \mathbf{3}$, macho, 15,6 mm; 4, fêmea, 17,3 mm; 5, fêmea, $14,3 \mathrm{~mm}$; 6, fêmea, 14,5 mm. 7-9. S. melanura: 7, macho, $12,1 \mathrm{~mm}$; 8 , fêmea, $15,3 \mathrm{~mm}$; 9, fêmea, $14,4 \mathrm{~mm}$. 

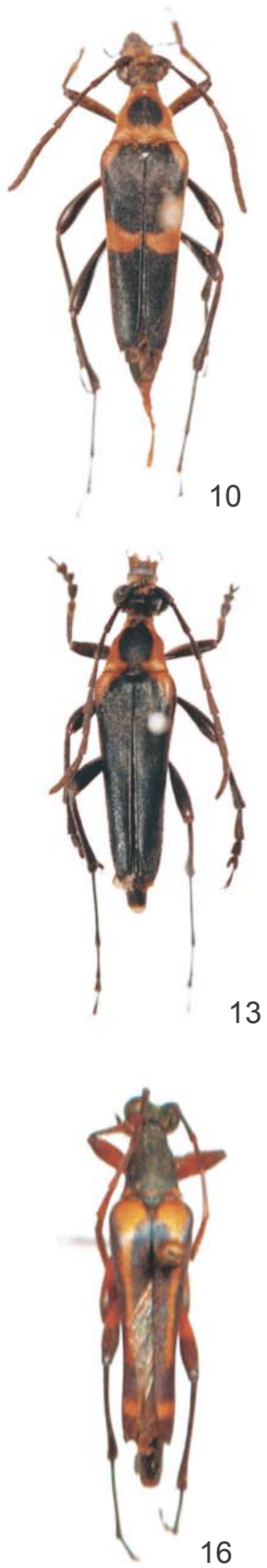
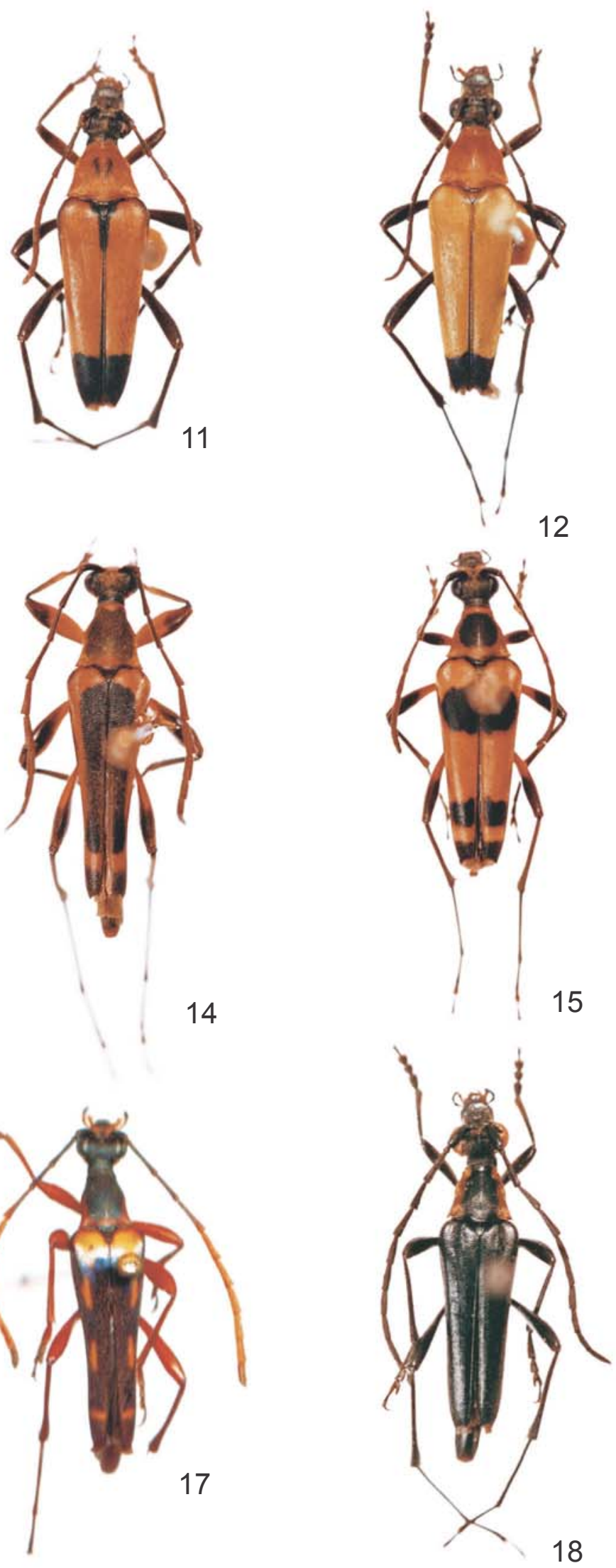

Figs. 10-18. 10-13, Strangalia succincta: 10, fêmea comprımento, 15,0 mm; 11, têmea, 14,6 mm; 12, fêmea, 13,4 mm; 13, macho, 16,1 mm. 14-15, S. lyrata: 14, macho, $13,7 \mathrm{~mm} ; \mathbf{1 5}$, fêmea, $11,8 \mathrm{~mm} . \mathbf{1 6 - 1 7}$, S. fulvicornis: 16, macho, $12,4 \mathrm{~mm} ; \mathbf{1 7}, \mathrm{macho}, 13,1 \mathrm{~mm} . \mathbf{1 8}, S$. melanophthisis: macho, $11,3 \mathrm{~mm}$. 
LinSLEY \& CHEMSAK (1971) sinonimizaram Ophistomis rustica com $O$. flavovittata. Examinamos os holótipos de $O$. flavovittata, O. variabilis e O. rustica (MZSP) e de $O$. fulvicornis (MNHN). Em ambos os sexos, variam a extensão das áreas ocupadas nos élitros por manchas pretas e amarelas (Figs 16, 17). As pernas podem ser pretas, amarelas ou bicolores e as antenas pretas ou parcialmente avermelhadas.

Material examinado. BRASIL. Minas Gerais: Virginia, fêmea (holótipo de O. rustica Melzer, 1926) (MZSP). Espirito Santo: macho (síntipo de O. fulvicornis Bates, 1872) (MNHN). Rio de Janeiro: Parque Nacional Itatiaia, macho. São Paulo: Peruibe, macho, fêmea (MNRJ); Santos, macho (holótipo de Ophistomis variabilis Melzer, 1926) (MZSP). São José Barreiro (Serra da Bocaina), 18 machos, fêmea; São Paulo (Cantareira), 4 fêmeas; (Jabaquara), 4 fêmeas; (Santo Amaro), fêmea; Teodoro Sampaio, 7 machos, 6 fêmeas (MNRJ). Paraná: Curitiba, 2 machos; Rio Negro, fêmea (holótipo de O. flavovittata Melzer, 1926) (MZSP). Santa Catarina: Corupá, 2 machos, 10 fêmeas; (Rio Vermelho), 8 machos, 7 fêmeas; Joinville, 2 fêmeas; Mafra, 46 machos, 28 fêmeas; Nova Teutonia, 68 machos, 11 fêmeas; Pinhal, 5 machos; Rio Natal, macho; São Bento do Sul, macho, fêmea (MNRJ). Rio Grande do Sul: Porto Alegre, macho; Santo Augusto, macho, 3 fêmeas; São Francisco de Paula, macho, fêmea (MNRJ). PARAGUAI. Itapuá: Itapuá, macho. Hohenau, fêmea (MNRJ). ARGENTINA. Misiones: Alto Paraná, macho, fêmea; Iguazú, macho. Leandro Alén, fêmea; Loreto, 5 machos, 2 fêmeas; San Pedro, macho (MNRJ). URUGUAI. Artigas: Sepulturas (Picada del Negro Muerto), 3 machos, 3 fêmeas (MNRJ).

\section{Strangalia melanophthisis (Berg, 1889) reval.}

(Fig. 18)

Euryptera melanophthisis Berg, 1889: 107; Bruch, 1912: 193 (cat.); Bosq \& Ruffinelli, 1951: 11 (distr.); Buck, 1959: 587 (distr.); Zajciw \& Ruffinelli, 1962: 18 (distr.); Zajciw \& Monné, 1968: 56 (distr.); Monné, 1995: 56 (cat.); Di Iorio, 1998: 143 (lect.); Cabrera \& Fernández, 2000: 37 (sintipos).

Strangalia melanophthisis; Di Iorio, 1998: 143 (in syn.). (= Euryptera dimidiata Redtenbacher, 1867)

Euryptera melanura var. nigripennis Melzer, 1930: 191; Zikán \& Wygodzinsky, 1948: 35 (tipo); Buck, 1959: 587 (distr.); Monné, 1995: 56 (cat.). Syn. nov.

Di IoRIO (1998: 143) considerou erroneamente Strangalia melanophthisis sinônimo de S. dimidiata (Redtenbacher, 1867), levando em consideração apenas a variação do colorido dos lados do protórax e da região anterior dos élitros (como observado anteriormente por BosQ \& RUfFinelLi 1951: 11), designou lectótipo para Euryptera melanophthisis (MACN) e transferiu-a para Strangalia.

MeLzer (1930: 190) ao descrever Euryptera melanura var. nigripennis, proveniente do Rio Grande do Sul, anotou que recebeu um macho, que descreveu como variedade, junto com outros recebidos de Porto Alegre e que "correspondem regularmente á descripção de E. melanophthisis Berg da Argentina e dahi vem a duvida bem fundamentada sobre a classificação definitiva, que podia ser resolvida com material da Republica visinha."

Di Iorio (1998) equivocou-se ao considerá-la como Strangalia melanura. O exame de material expressivo comprova a variabilidade do colorido.
Material examinado. BRASIL. Mato Grosso do Sul: Campo Grande, macho. São Paulo: Teodoro Sampaio, 2 machos. Paraná: Arapongas, 2 machos; Caviuna, 2 machos; Guarapuava, macho; Ponta Grossa, macho; Rolandia, 3 machos. Santa Catarina: Nova Teutonia, 19 machos, fêmea. Rio Grande do Sul: Esteio, 2 machos, 2 fêmeas; Parecy Novo, macho; Pelotas, 3 machos, 2 fêmeas. PARAGUAI, Guairá: Villarrica, fêmea. Itapuá: Itapuá, 2 machos. ARGENTINA. Misiones: Alto Paraná, fêmea; Loreto, 7 machos. Entre Ríos: Liebig, macho. URUGUAI. Tacuarembó: Puntas del Arroyo Laureles, 2 machos. Rocha: Parque San Miguel, macho. San José: Paso Pache (Rio Santa Lucía), 2 fêmeas. (MNRJ).

\section{Anastrangalia sanguinolenta (Linnaeus, 1761)}

Leptura sanguinolenta Linnaeus, 1761: 196; Reitter, 1912: 18; Aurivillius, 1912: 217 (cat.).

Anoplodera sanguinolenta; Gressitt, 1951: 85.

Anastrangalia sanguinolenta; Villiers, 1978: 216.

Leptura variabilis DeGeer, 1775: 137.

Leptura bonaeriensis Burmeister, 1865: 177; Bachmann \& Di Iorio, 2002: 61 (tipo). Syn. nov.

Ophiostomis [sic] bonariensis; Bruch, 1912: 193 (cat.).

? Leptura bonaerensis; Monné \& Giesbert, 1995: 170.

"Leptura" bonariensis; Di Iorio, 1998: 140.

Espécie amplamente distribuída na região Paleártica. Introduzida na Argentina, um exemplar coletado em Buenos Aires e identificado por J. M. Bosq como Leptura bonaeriensis (MNRJ) possibilitou a presente sinonímia. No início da descrição original BURMEISTER (1865) comparou, pelo aspecto e dimensões, $L$. bonaeriensis com L. sanguinolenta.

Material examinado. ARGENTINA. Buenos Aires: Ciudad, fêmea, 1.XI.1913, J. M. Bosq col. (identificada por J. M.Bosq como $L$. bonaeriensis). ALEMANHA. Bayern: Eggenthal, 7 machos, 3 fêmeas. Mittenwald, fêmea. ITALIA. Veneto: Belluno, macho, 2 fêmeas. Friuli Veneza Giulia: Trieste, fêmea. (MNRJ).

\section{REFERÊNCIAS}

Aurivillius, C. 1912. Coleopterorum Catalogus, pars 39, Cerambycidae: Cerambycinae. Berlin, W. Junk, 574 p.

Bachmann A. O. \& O. Di Iorio. 2002. Types and related specimens of Cerambycidae and Disteniidae (Coleoptera) in the Museu Argentino de Ciencias Naturales "Bernardino Rivadavia", Buenos Aires, Argentina. Revista del Museo Argentino de Ciencias Naturales n. s. 4(1): 55-93.

Bates, H. W. 1872. On the longicorn Coleoptera of Chontales, Nicaragua. Transactions of the Entomological Society of London 1872 : $163-238$.

Baucke, O. 1957. Cerambicídeos do Rio Grande do Sul. Iheringia 8: 130 .

Belon, P. M. 1897. Remarques sur le genre Euryptera Serv. du groupe des lepturides et description d'une espéce nouvelle de Bolivie. Annales de la Société Entomologique de Belgique 41: 339-342.

Berg, F. G. C. 1889. Quadraginta Coleoptera nova Argentina. Anales de la Universidad de Buenos Aires 6: 105-157.

Bosq, J. M. \& A. Ruffinelli. 1951. Notas para el catálogo de los Cerambícidos del Uruguay. Comunicaciones Zoológicas del Museo de Historia Natural 3(62): 1-32.

Bruch, C. 1912. Catálogo sistemático de los Coleópteros de la República Argentina. Pars VIII. Família Cerambycidae. Revista del Museo 
de La Plata 18: 179-226.

Buck, P. 1959. Cerambycidae in der Sammlung des Instituto Anchietano de Pesquisas. Pesquisas 3: 577-609.

Burmeister, H. C. 1865. Longicornia Argentina. Systematische Uebersicht der Bockkäfer der La Plata-Staaten. Stettiner Entomologische Zeitung 26: 156-181.

Cabrera, N. \& L. A. Fernández. 2000. Los ejemplares tipo de Cerambycidae del Museu de La Plata (Insecta, Coleoptera). Revista del Museo de La Plata, Série Técnica y Didáctica, 38: 33-38.

DeGeer, C. 1775. Mémoires pour servir à l'histoire des insectes. Stockholm, Pierre Hesselberg, 5: vii +448 p.

Di IoRIO, O. 1998. Redescriptions, new combinations, synonimies, and new records of South American Lepturini (Coleoptera, Cerambycidae, Lepturinae). Insecta Mundi 12 (1-2): 139-148.

Gounelle, E. 1911. Liste des cérambycides de la région de Jatahy, Etat de Goyaz, Brésil.Annales de la Société Entomologique de France 80: 1-150.

Gressitt, J. L. 1951. Longicorn beetles of China. Longicornia 2: 1667.

Horn, W. \& I. Kahle. 1936. Über entomologische Sammlungen. Entomologische Beiheft aus Berlin-Dahlem 2: 161-296.

Lacordaire, J. T. 1869. Histoire Naturelle des Insectes. Genera des Coléoptères, ou exposé méthodique et critique de tous les genres proposés jusqu'ici dans cet ordre d'insectes. Paris, Roret, 8: 1-552.

Linnaeus, C. 1761. Fauna Suecica sistens animalia Sueciae regni: Mammalia, Aves, Amphibia, Pisces, Insecta, Vermes. Distributa per classes et ordines, genera et species,cum differentiis specierum, synonymis auctorum, nominibus incolarum, locis natalium, descriptionibus insectorum. Holmiae, Salvius, 578 p.

Linsley, E. G. \& J. A. Chemsak. 1971. An attempt to clarify the generic status of some Neotropical species currently assigned to Euryptera, Chontalia and Ophistomis (Coleoptera, Cerambycidae). Arquivos de Zoologia 21(1): 1-40.

Meске, R. 2002. Insetos do Pinheiro brasileiro - Insekten der brasilianischen Araukarie - Insects of the Brasilian Pine. Tübingen, Attempto Service GmbH, 79 p.

Mecke, R.; M. H. M. Galileo \& W. Engels. 2000. Insetos e ácaros associados à Araucaria angustifolia (Araucariaceae, Coniferae) no sul do Brasil. Iheringia, Série Zoologia, 88: 165-172.

Melzer, J. 1922. Longicórneos (Col.) do Brasil, novos ou pouco conhecidos. Notas Preliminares do Museu Paulista 2(2): 112 .

Melzer, J. 1926. Longicórneos (Col.) do Brasil, novos ou pouco conhecidos. Publicações do Museu Nacional 7: 1-15.

Melzer, J. 1927. Longicórneos (Col.) do Brasil, novos ou pouco conhecidos. Revista do Museu Paulista 15(1): 135-202.

Melzer, J. 1930. Longicórneos do Brasil, novos ou pouco conhecidos II (Coleoptera, Cerambycidae). Archivos do Instituto Biológico 3: $187-208$.

Monné, M. A. 1995. Catalogue of the Cerambycidae (Coleoptera) of the Western Hemisphere. Part XXI. Subfamily Lepturinae. São Paulo, Sociedade Brasileira de Entomologia, 159 p.

Monné, M. A. 2002. Catalogue of the Neotropical Cerambycidae
(Coleoptera) with known host plant - Part V: Subfamilies Prioninae, Parandrinae, Oxypeltinae, Anoplodermatinae, Aseminae and Lepturinae. Publicações Avulsas do Museu Nacional 96: 1-70.

Monné, M. A. \& E. F. Giesbert. 1995. Checklist of the Cerambycidae and Disteniidae (Coleoptera) of the Western Hemisphere. Burbank, Wolfsgarden Books, xiv +419 p.

Monné, M. A. \& D. Zajciw. 1970. Cerambícidos del Uruguay, nuevos o poco conocidos II. Atas da Sociedade de Biologia 13(1-2): 2932 .

Redtenbacher, L. 1867. Reise des österreichischen Fregatte Novara und die Erde in den Jahren 1857, 1858, 1859, unter der befehlen des Commodore B. von Wüllerstorf- Urbair. Wien, Zoologischer Theil. Zweiter Band: Coleopteren, 249 p.

Reitter, E. 1912. Fauna Germanica. Die Käfer des Deutschen Reiches. Stuttgart, K. G. Lutz' Verlag, 4: 1-236.

Thomson, J. 1860. Essai d'une classification de la famille des cérambycides et matériaux pour servir a une monographie de cette famille. Paris, Bouchard-Huzard, $404 \mathrm{p}$.

Thomson, J. 1864. Systema cerambycidarum ou exposé de tous les genres compris dans la famille des cérambycides et familles limitrophes. Mémoires de la Société Royal de Sciences de Liège 19: 1540 .

Thomson, J. 1878. Typi cerambycidarum Musei Thomsoniani. Paris, E. Deyrolle, $21 \mathrm{p}$.

Villiers, A. 1978. Faune des Coléoptères de France I. Cerambycidae. Encyclopedie Entomologique 1978: xxvii + 611 p.

Zasciw, D. 1958. Fauna do Distrito Federal XLVIII. Contribuição para o conhecimento dos longicórneos de Rio de Janeiro (Coleoptera, Cerambycidae). Boletim do Museu Nacional, Zoologia, 189: 126, 2 figs.

Zajciw, D. 1970. Descrição do macho e redescrição da fêmea de Ophistomis flavocincta Thomson, 1860, com observações sôbre o desenho dos élitros (Coleoptera, Cerambycidae, Lepturini). Anais da Academia Brasileira de Ciências 42(2): 229-233.

ZAJCIW, D. 1972. Contribuição para o estudo da fauna dos longicórneos do Parque Nacional do Itatiaia (Coleoptera, Cerambycidae). Brasil Florestal 3: 40-72.

ZAJCIW, D. 1974. Contribuição para o estudo da fauna dos longicórneos (Coleoptera, Cerambycidae) das florestas do Estado do Espírito Santo e principalmente da Reserva Biológica Soôretama. Boletim Técnico do Instituto Brasileiro de Desenvolvimento Florestal 4: 37-91.

Zajciw, D. \& M. A. Monné. 1968. Cerambícidos del Uruguay, nuevos o poco conocidos. Revista de la Sociedad Uruguaya de Entomologia 7: 51-61.

Zajciw, D. \& A. Ruffinelli. 1962. Fauna de los Cerambícidos del Uruguay. Boletin de la Facultad de Agronomia 60: 1-89.

Zajciw, D. \& C. A. C. Seabra. 1968. Longicórneos da Serra da Bocaina, Estado de São Paulo (Coleoptera, Cerambycidae). Atas da Sociedade de Biologia 12(2): 69-72.

ZIKÁN, J. F. \& W. ZIKÁN. 1944. A inseto-fauna do Itatiaia e da Mantiqueira. Boletim do Ministerio de Agricultura 33(8): 1-50.

Zikán, W. \& P. WygodZinsky. 1948. Catálogo dos tipos de insetos do Instituto de Ecologia e Experimentação Agrícolas. Boletim do Serviço de Pesquisas Agronômicas 4: 1-93. 